\title{
Réflexions et propositions pour le calcul optimisé des parois clouées
}

\author{
Marc Favre ${ }^{\star}$, Éric Vermoote et Damien Fuentes \\ GEOS ingénieurs conseils, 310, avenue Marie-Curie, bâtiment Europa 2, Archamps Technopole, 74160 Archamps, France
}

\begin{abstract}
Résumé - Cet article a pour objets : (1) de présenter les problèmes auxquels sont confrontés les praticiens lors du dimensionnement des parois clouées en particulier pour l'optimisation du quantitatif d'inclusions et de la détermination des efforts apportés au parement ; (2) à partir de développements ou extensions de méthodes existantes, de proposer des méthodes permettant d'optimiser les longueurs et sections des inclusions et de déterminer les efforts ponctuels apportés au parement par les inclusions afin de pouvoir réaliser le dimensionnement structurel de ce dernier ; (3) de présenter les développements opérationnels résultant de ces développements. La méthode proposée est basée, entre autres, sur des développements de propositions contenues dans l'additif aux recommandations CLOUTERRE publié en 2002 et dans la norme NF 94270 publiée en 2009. Une réflexion a été menée sur le mécanisme de transfert de l'effort mobilisé à l'intersection entre l'inclusion et la surface de rupture jusqu'au parement pour déterminer les efforts ponctuels appliqués par les têtes d'inclusions à ce dernier.
\end{abstract}

Mots clés : parois clouées / inclusions / parement / méthode de Culmann / poussée active et au repos

\begin{abstract}
Proposals for the design optimization of nailed walls. The goals of this document are: (1) to present the problems encountered by practitioners when making the design of soil nail walls, particularly to optimize the quantity of nail bars and to determine the efforts applied to the shotcrete wall facing; (2) to propose methods giving answers to the above-mentioned problems by development of existing methods; (3) to present the practical solutions. The method is based on developments of the proposals included in the French document "Addendum to the CLOUTERRE recommendations" published in 2002 and in the French standard NF 94270 geotechnical design-retaining structuresreinforced and soil nailing structures published in 2009. The point loads due to nail heads applied to the shotcrete face are determined from the efforts in the nail bars taking into account the mechanism of transfer.
\end{abstract}

Keywords: nailed walls / nail bars / shotcrete wall facing / Culmann's method / active and at rest pressure

\section{Préambule}

Le dimensionnement des ouvrages de soutènement du type paroi clouée en appliquant l'Eurocode 7 (Vezole et al., 2009) pose aux praticiens un certain nombre de problèmes :

- pour les vérifications géotechniques GEO :

- le calcul des longueurs de clous strictement nécessaires pour satisfaire les critères de stabilité ;

- pour les vérifications structurales STR :

- le calcul des sections d'acier strictement nécessaires dans les inclusions,

- le calcul des efforts ponctuels appliqués au parement par les têtes d'inclusions et/ou de la répartition de la pression de contact sol-parement.

$\bar{\star}$ Auteur de correspondance : marc.favre@geos.fr
Le calcul des longueurs de clous strictement nécessaires implique que le concepteur suppose un maillage initial de clous (espacements vertical et horizontal) et la longueur de ces derniers. Sur cette base sont réalisés des calculs de vérification qui conduisent à allonger ou raccourcir les lits de clous suivant que les facteurs de sécurité calculés sont respectivement insuffisants ou surabondants. Cette démarche, où le diamètre de forage et le frottement latéral unitaire caractérisant l'interaction limite sol-clou sont fixés, requiert que le concepteur procède par tâtonnements. Par ailleurs, cette démarche ne garantit pas que la quantité de clous soit minimisée.

Les algorithmes de calcul utilisés jusqu'à ce jour calculent la résistance mobilisable à l'arrière de la surface de rupture (minimum de la résistance structurale et de la résistance à l'arrachement) puis intègrent cet effort dans les équations d'équilibre pour calculer le facteur de sécurité mais ces algorithmes ne fournissent pas l'effort strictement nécessaire 
dans chaque inclusion pour atteindre le facteur de sécurité visé : le dimensionnement de la section des inclusions doit donc être réalisé lui aussi par tâtonnements.

Enfin, les algorithmes et méthodes de calculs actuels ne fournissent pas les efforts ponctuels appliqués au parement.

Le but de cet article est de proposer une méthode de calcul permettant de répondre à ces problèmes et de présenter l'implémentation qui en a été faite dans un logiciel.

\section{Analyse des documents de références}

Les référentiels applicables au dimensionnement des parois clouées ont évolué :

- recommandations CLOUTERRE (1991) ;

- additif 2002 aux recommandations CLOUTERRE (2002) ;

- norme NF P 94-270 sur les ouvrages en sol renforcé (Vezole et al., 2009).

Ce dernier document s'avère être, à l'usage, difficilement applicable pour le praticien, puisqu'il propose dans son annexe E3 :

- soit une méthode simplifiée applicable seulement pour des cas « simplistes »;

- soit par une modélisation numérique sans que cette dernière soit détaillée.

Compte tenu des conditions particulièrement restrictives pour que puisse être appliquée la méthode "simplifiée », les cas de la pratique courante relèveraient tous de la modélisation numérique qui reste peu explicite, ce qui conduit, par la suite, à réflexions et propositions.

La modélisation simplifiée recommandée consiste à déterminer la valeur de l'effort de traction maximal dans chaque lit de renforcement :

- à partir de la valeur de la résultante $P_{0 ; \mathrm{d}}$ de la poussée au repos s'exerçant, avant le début de l'excavation, sur un écran vertical positionné au voisinage du parement et de même hauteur que celui-ci ;

- en distribuant cet effort $P_{0 ; \mathrm{d}}$ dans les lits de clous pour tenir compte des conséquences des phases de construction qui conduisent à une augmentation des efforts en tête et à une réduction en pied.

Dans la pratique, la distribution conduisant à une résultante d'intensité $P_{0 ; \mathrm{d}}$ est obtenue en ajoutant une pression uniforme $\sigma_{0 ; \mathrm{d}}$ à la pression $\sigma_{\mathrm{ah;d}}$ qui représente la composante horizontale de la poussée active selon Rankine (Fig. 1).

Avec :

$-P_{0 ; \mathrm{d}}$ : valeur de calcul de la poussée des terres au repos;

$-\sigma_{0 ; \mathrm{d}}$ : valeur de calcul de la contrainte horizontale uniforme à ajouter à $\sigma_{\mathrm{ah} ; \mathrm{d}}$.

$$
\sigma_{0 ; \mathrm{d}}=\frac{1}{2}\left(K_{0}-K_{a}\right) \gamma H .
$$

$-K_{0}$ : coefficient de poussée des terres au repos;

$-K_{\mathrm{a}}$ : coefficient de poussée active des terres ;

$-\sigma_{\mathrm{ah} ; \mathrm{d}}$ : valeur de calcul de la composante horizontale de la poussée active selon Rankine.

Dans ces conditions, il est apparu intéressant que soit développée et proposée une méthode pour les cas qui relèvent de la pratique courante.

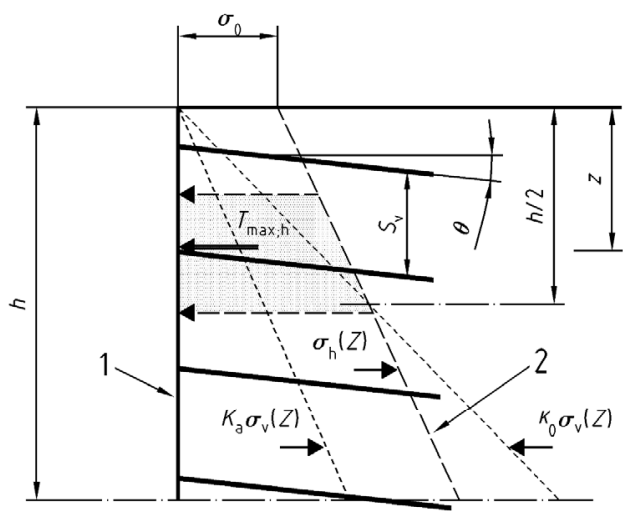

Fig. 1. Distribution des efforts à considérer dans les lits de clous pour vérifier la stabilité interne d'un massif cloué dans le cas d'une modélisation simplifiée (Vezole et al., 2009). 1 : écran vertical positionné au voisinage du parement ; 2 : distribution des contraintes horizontales à considérer.

Fig. 1. Diagram of pressure to be taken into account to check the internal stability of a nailed wall (Vezole et al., 2009).

\section{Problématique des calculs}

\subsection{Calcul des efforts appliqués au parement}

Dans la pratique courante, la problématique du calcul des efforts appliqués au parement et de son dimensionnement reste entière.

\subsubsection{Généralités}

En accord avec l'additif 2002 aux recommandations CLOUTERRE, 1991 (CLOUTERRE, 2002), l'effort ponctuel retenu en tête de clou pour dimensionner le parement est :

$$
T_{N}=\max \left[T_{0} ; T_{1} ; T_{2}\right] \cos (\theta-\eta),
$$

avec :

$-\theta$ : angle de l'axe du clou sous l'horizontale ;

$-\eta$ : angle d'inclinaison du parement par rapport à la verticale ;

- $T_{0}$ : calculé à partir de $T_{\text {MAX }}$ permettant de justifier la stabilité d'ensemble ;

- $T_{1}$ : calculé afin d'assurer la stabilité du parement par frottement ;

$-T_{2}$ : calculé pour assurer le confinement;

- $T_{\text {MAX }}$ : borne supérieure ou enveloppe des tractions dans les clous permettant de justifier la stabilité d'ensemble dans tous les cas de figure des calculs.

\subsubsection{Calcul de $T_{\text {MAX }}$}

Le principe de calcul est le suivant :

- pour chaque surface de rupture $S_{\mathrm{i}}$ envisagée :

- faire un calcul du facteur de sécurité, $F_{\text {oi }}$, sans inclusions,

- faire un calcul du facteur de sécurité, $F_{\text {ri }}$, avec inclusions dont les longueurs sont saisies initialement par l'utilisateur mais sans en limiter la résistance structurale,

- connaissant l'effort stabilisant, $T_{\mathrm{io}}$, apporté par chaque inclusion sur la surface de rupture $S_{\mathrm{i}}$ et sachant que l'effort stabilisant global, $A_{\mathrm{i}}$, apporté par l'ensemble des 


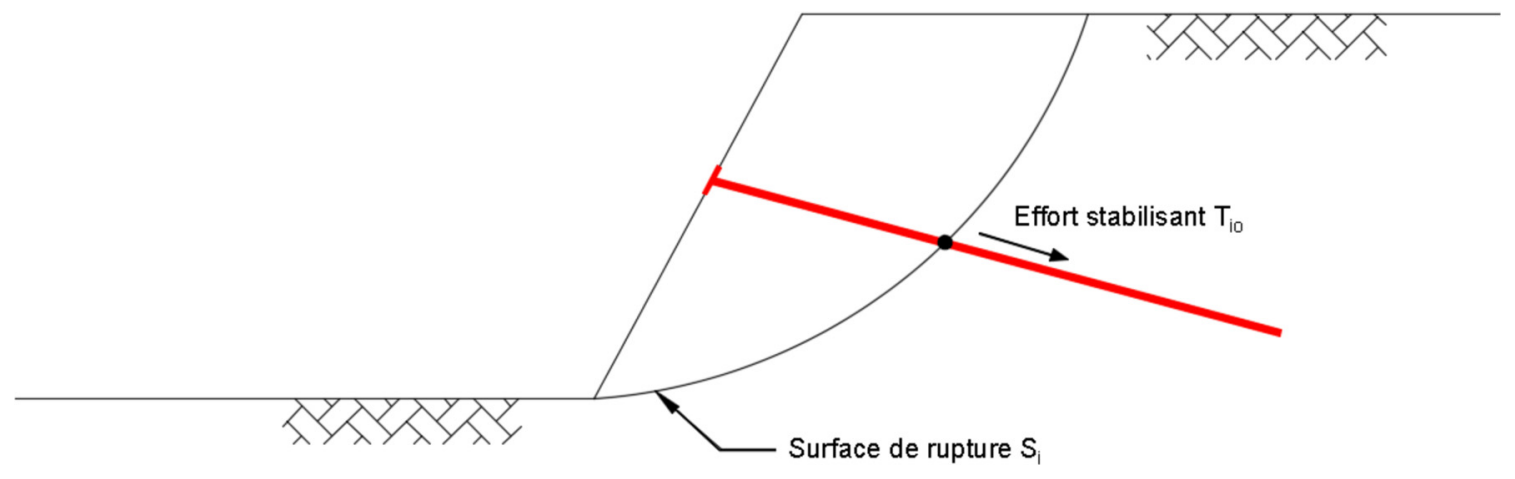

Fig. 2. Position du problème du calcul itératif de l'effort $T_{\mathrm{i}}$ dans une inclusion pour assurer la stabilisation interne suivant la surface de rupture $S_{\mathrm{i}}$. Fig. 2. Definition of the problem of the iterative calculation of the force, $T_{i}$, in the nail bar to ensure the internal stabilization along the failure surface $S_{i}$.

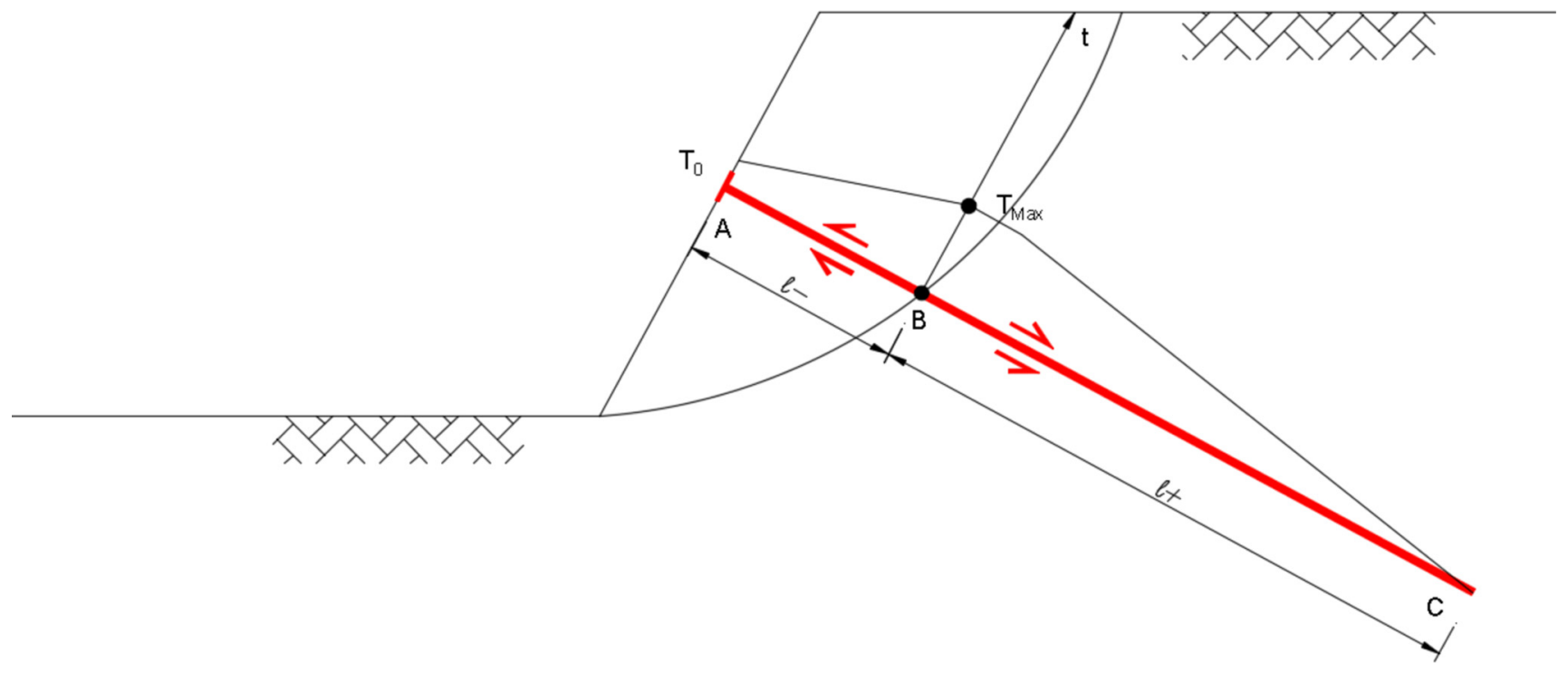

Fig. 3. Variation des efforts le long de l'inclusion.

Fig. 3. Variation of the forces along the nails.

clous, fait passer le facteur de sécurité $F_{\mathrm{oi}}$ à $F_{\mathrm{ri}}\left(\geq F_{\mathrm{oi}}\right)$, il est recherché par itérations les valeurs des efforts $T_{\mathrm{i}}$ strictement nécessaires pour que $F_{\mathrm{ri}} \geq F$ requis (Fig. 2).

Avec :

$-F_{\mathrm{oi}}$ : facteur de sécurité de la surface de rupture sans inclusion(s) ;

$-F_{\text {ri }}$ : facteur de sécurité de la surface de rupture avec inclusion(s) ;

$$
\text { avec l'effort } T_{\mathrm{io}}, F_{\mathrm{ri}}=F_{\mathrm{oi}}+\Delta F_{\mathrm{TiO}} \text {. }
$$

Le problème consiste :

- à rechercher $T_{\mathrm{i}}$ tel que : $\Delta F_{\mathrm{T}_{\mathrm{i}}}=F_{\text {visé }}-F_{\text {oi }}(3)$;

- à itérer les calculs ci-dessus pour toutes les surfaces de rupture et pour toutes les phases afin d'obtenir $T_{\mathrm{MAX}}=$ $\max \left[T_{\mathrm{i}}\right]$.

Cette méthode peut être appliquée en considérant que les inclusions travaillent soit de manière axiale soit selon l'approche multicritère telle que proposée par Blondeau et al. (1984).

\subsubsection{Détermination de $T_{\mathrm{o}}$ à partir de $T_{\mathrm{MAX}}$}

L'optimisation proposée suppose que la longueur des clous est toujours conditionnée par $T_{\mathrm{MAX}}$.

Il est proposé que $T_{\mathrm{o}}$, effort amené au parement pour assurer la stabilité globale, soit déduit de $T_{\mathrm{MAX}}$ en retranchant de ce dernier le frottement mobilisé entre la surface de rupture et le parement.

À l'extrême, si le clou est gainé et peut donc coulisser librement dans la zone entre la surface de rupture et le parement, ce qui correspondrait à ce qui se passe sur la longueur libre d'un tirant actif, alors $T_{\mathrm{o}}=T_{\mathrm{MAX}}$.

À l'inverse, si l'effort de frottement mobilisable est supérieur à $T_{\mathrm{MAX}}$ dans cette zone du fait d'un frottement latéral unitaire limite et/ou d'une longueur passive du clou élevé, l'effort $T_{\mathrm{MAX}}$ est complètement transmis au sol avant même d'atteindre le parement et $T_{\mathrm{o}}=0$.

La représentation du mécanisme est celle présentée sur la Figure 3.

$$
\text { où } T_{\mathrm{MAX}}=\min \left[\frac{T_{\text {barre }}}{\Gamma_{\mathrm{acier}}} ; \pi \varphi_{\mathrm{f}} l^{+} \frac{q_{\mathrm{s}_{\mathrm{BC}}}}{\Gamma_{\mathrm{q}_{\mathrm{s}}}}\right] \text {, }
$$




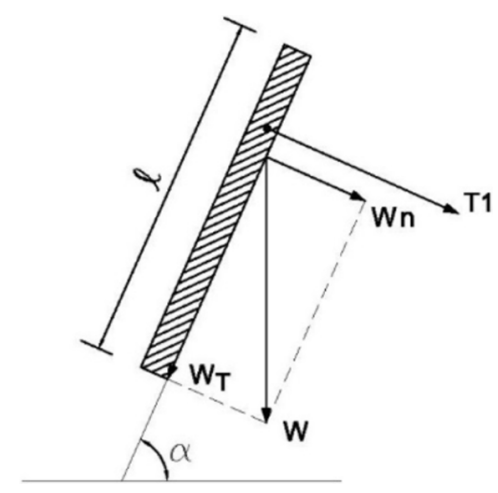

Fig. 4. Modélisation des efforts de glissement de la paroi.

Fig. 4. Modelling of the sliding forces of the wall.

$$
\text { et } T_{o}=\max \left(T_{\mathrm{MAX}}-\pi \varphi_{\mathrm{f}} l^{-} \frac{q_{\mathrm{s}_{\mathrm{AB}}}}{\Gamma_{\mathrm{q}_{\mathrm{s}}}} ; 0\right) \text {, }
$$

avec :

$-\Gamma_{\text {acier }}$ : le facteur de sécurité partiel sur l'acier ;

$-\varphi_{\mathrm{f}}$ : le diamètre de forage du clou ;

$-q_{\mathrm{s}}$ : le frottement latéral unitaire du sol ;

$-\Gamma_{\mathrm{q}_{\mathrm{s}}}$ : le facteur de sécurité partiel sur le frottement latéral unitaire.

\subsubsection{Calcul de $T_{1}$}

$T_{1}$ est l'effort nécessaire pour mobiliser un frottement suffisant à l'arrière du parement afin d'éviter les mouvements tangentiels.

La Figure 4 précise les données du problème. Il faut néanmoins noter que la résolution présentée ci-dessous est simplifiée, car elle ne tient pas compte de l'angle entre le parement et le clou.

$$
\begin{aligned}
& F_{\text {moteur }}=W_{T}=W \sin \alpha \\
& F_{\text {Résistant }}=\left(W_{N}+T_{1}\right) \tan \varphi_{\mathrm{i}}+c_{\mathrm{i}} l \\
& F_{\text {Résistant }}=\left(W \cos \alpha+T_{1}\right) \tan \varphi_{\mathrm{i}}+c_{\mathrm{i}} l \\
& F_{G}=\frac{F_{\text {Res }}}{F_{M o t}}=\frac{W \cos \alpha \tan \varphi_{\mathrm{i}}+T_{1} \tan \varphi+c_{\mathrm{i}} l}{W \sin \alpha}, \\
& T_{1}=\left(F_{G} W \sin \alpha-W \cos \alpha \tan \varphi_{\mathrm{i}}-c_{\mathrm{i}} l\right) \times \frac{1}{\tan \varphi_{i}}, \\
& T_{1}=W\left(F_{G} \frac{\sin \alpha}{\tan \varphi_{i}}-\cos \alpha\right)-\frac{c_{i} l}{\tan \varphi_{i}},
\end{aligned}
$$

avec :

$-W=25\left(\mathrm{kN} / \mathrm{m}^{3}\right) \times e(m) \times 1(m)$ pour un espacement de $1 \mathrm{~m}$ dans la troisième direction;

$-\varphi_{\mathrm{i}}=$ angle de frottement à l'interface sol-parement ;

$-c_{\mathrm{i}}=$ cohésion à l'interface sol-parement ;

$-e=$ épaisseur du parement.

L'effort $T_{1}$ est distribué entre les clous au prorata des surfaces reprises par chacun d'eux.

\subsubsection{Calcul de $T_{2}$ \\ 3.1.5.1 Généralités}

La méthode pour estimer $T_{2}$ est une extension de la méthode simplifiée proposée dans l'annexe E3 de la norme NF 94270 (Vezole et al., 2009) et consiste, dans un premier temps,

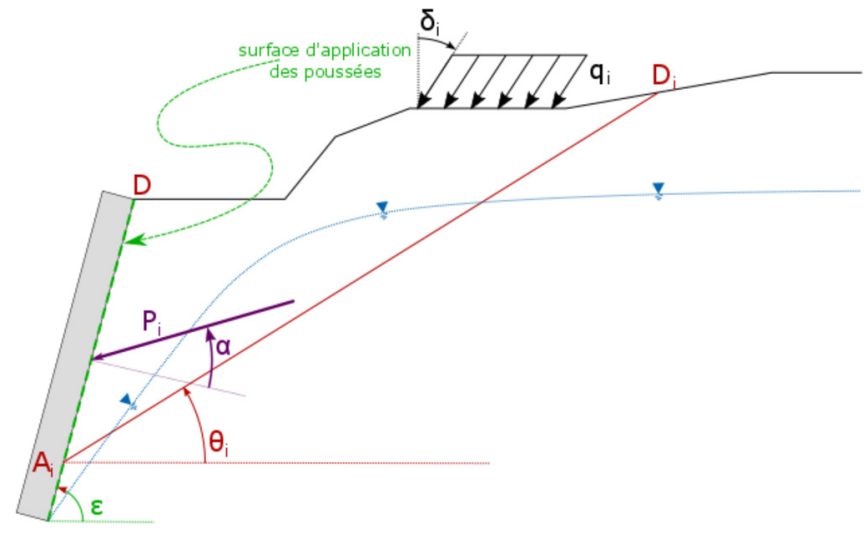

Fig. 5. Détermination de la résultante des poussées.

Fig. 5. Calculation of the active earth pressure.

à calculer la poussée active à l'arrière du parement considéré comme un écran fictif par la méthode de Culmann.

$T_{2}$ est l'effort de confinement apporté par les clous qui garantit la stabilité vis-à-vis des efforts de poussée active sur le parement.

\subsubsection{Méthodologie générale d'obtention du diagramme des pressions et des résultantes des poussées}

Le calcul de chacun des diagrammes de pressions (sol, eau et surcharges) est réalisé suivant le même principe :

- le parement sur lequel s'exercent les poussées est discrétisé suivant un pas choisi par l'utilisateur : cette discrétisation génère les points $A_{\mathrm{i}}$ définis sur la Figure 5 ;

- les incréments de poussée s'exerçant sur le parement sur chaque segment de discrétisation sont calculés par la méthode de Culmann ;

- la répartition des résultantes des poussées sur le parement permet de calculer le diagramme des poussées, traduites en pression sur le parement, par dérivation du diagramme des forces ;

- la résultante de la poussée totale est ensuite calculée par sommation des incréments de poussée.

La méthode de Culmann est utilisée pour la détermination des poussées et permet de tenir compte d'une forme quelconque de terre-plein à l'amont du mur et d'une surcharge elle aussi quelconque.

Le point d'application de la poussée est donné par le barycentre du diagramme de pressions déterminé ci-dessus.

C'est une généralisation de la théorie de Coulomb, puisque basée sur des lignes de rupture rectilignes.

Comme le recommande Tschebotarioff (1968), le frottement sol-parement peut être négligé $(\alpha=0)$ dans le calcul de la poussée qui sera alors légèrement surestimée de 5 à $10 \%$.

Les forces s'exerçant sur le prisme de terre $A D D_{\mathrm{i}}$ sont les suivantes, par mètre linéaire (Fig. 6) :

- $W_{\mathrm{i}}$ : poids des terres du prisme $A D D_{\mathrm{i}}$ pour une épaisseur de $1 \mathrm{~m}$;

$-W_{\mathrm{i}}:$ aire $\left(A D D_{\mathrm{i}}\right) \times \gamma$;

- $Q_{\mathrm{i}}$ : résultante des surcharges sur le prisme $A D D_{\mathrm{i}}, Q_{\mathrm{i}}=\int q_{\mathrm{i}}$;

- $U_{\mathrm{i}}$ : résultante des pressions interstitielles sur le plan $A D_{\mathrm{i}}$;

- $C_{\mathrm{i}}$ : cohésion sur le plan $A D_{\mathrm{i}} C_{\mathrm{i}}=C \times l\left(A D_{\mathrm{i}}\right)=C \times l_{\mathrm{i}}$ pour un sol de cohésion homogène ; 


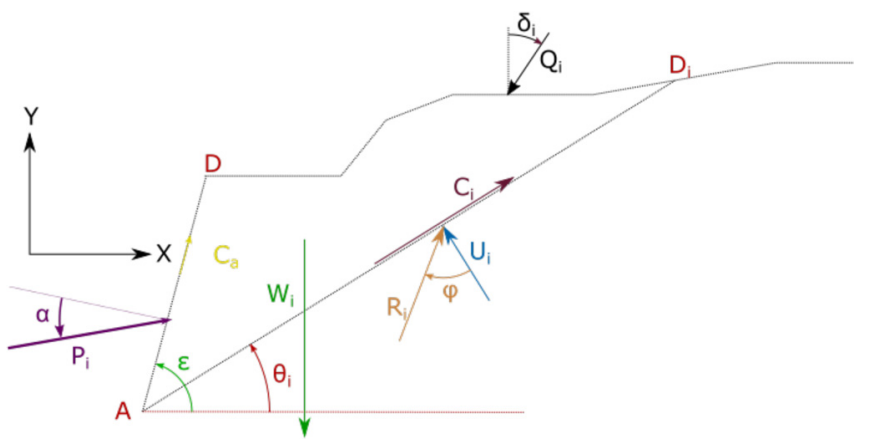

Fig. 6. Forces s'exerçant sur le prisme de terre.

Fig. 6. Forces acting on the earth block.

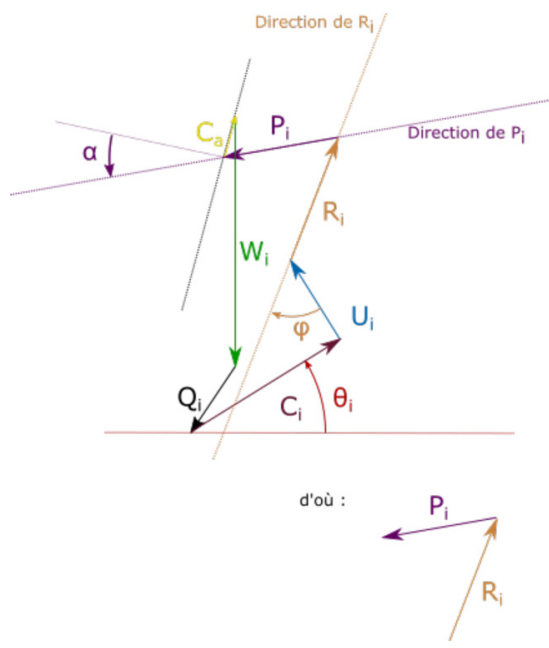

Fig. 7. Polygone des forces.

Fig. 7. Polygon of forces.

- $C a_{\mathrm{i}}$ : adhérence sur le parement $C a_{\mathrm{i}}=C a \times l(A D)$;

- $R_{\mathrm{i}}$ : réaction du plan $A D_{\mathrm{i}}$ de valeur inconnue mais inclinée de $\varphi$ par rapport à la normale au plan $A D_{\mathrm{i}}$;

$-P_{\mathrm{i}}$ : résultante des poussées de valeur inconnue mais inclinée de $\alpha$ s'exerçant sur le plan $A D$ en considérant le plan de rupture $A D_{\mathrm{i}}$ pour une épaisseur de $1 \mathrm{~m}$;

$-\alpha$ : inclinaison de la poussée active sur le parement fixée par le concepteur (Fig. 7).

La conduite de ce calcul pour les plans $A D_{1}$ à $A D_{\mathrm{n}}$, inclinés de $\theta_{\mathrm{i}}$ à $\theta_{\mathrm{n}}$, sur l'horizontale permet de déterminer l'évolution de la poussée $P_{\mathrm{i}}$ sur le parement en fonction de l'inclinaison du plan de rupture considéré.

La méthode de Culmann donne la résultante totale des poussées sur le parement jusqu'au point $\mathrm{A}$.

Afin de pouvoir tracer le diagramme des pressions s'exerçant sur le parement, il y a lieu de recommencer le calcul de la résultante totale des poussées pour différentes positions du point $\mathrm{A}$ sur le parement. Les différences de poussées entre deux positions consécutives permettent alors de déterminer le diagramme des poussées sous forme de pression sur le parement (Fig. 8).

Soit :

$-\sigma_{\mathrm{j}}$ : la pression au point $A_{\mathrm{j}}$;

$-\sigma_{\mathrm{j}-1}$ : la pression au point $A_{\mathrm{j}-1}$;

$-\Delta_{\text {inc }}$ : la distance entre deux points de discrétisation;

$-\sigma_{\mathrm{j}}$ est donné par : $\sigma_{j}=\frac{P_{j}-P_{\mathrm{j}-1}}{\Delta_{\text {inc }}}$.

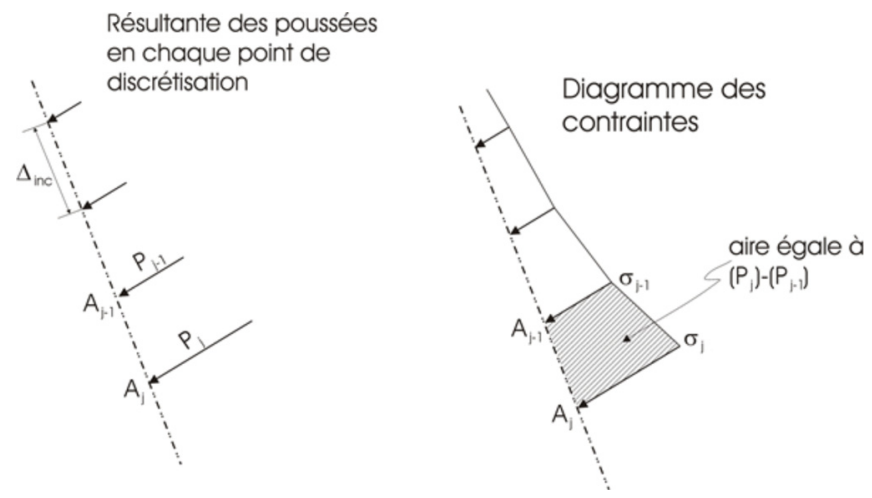

Fig. 8. Calcul des diagrammes de pression sur le parement.

Fig. 8. Determination of the pressure diagrams on a wall.

\subsubsection{Poussée au repos ou poussée active - comparaison en efforts horizontaux}

Pour les valeurs courantes de $\varphi$, c'est-à-dire comprises entre $25^{\circ}$ et $40^{\circ}$, le rapport $K_{\mathrm{o}} / K_{\mathrm{a}}$ (avec $K_{\mathrm{o}}$ le coefficient de poussée des terres au repos déterminable à l'aide de la formule de Jaky et $K_{\mathrm{a}}$ le coefficient de poussée active des terres) égale en première approximation à $(1-\sin \varphi) / \tan ^{2}\left(\frac{\pi}{4}-\frac{\varphi}{2}\right)$ pour une contrainte horizontale comprise entre 1,42 et 1,64 , soit environ 1,5 pour un angle de frottement courant de $30^{\circ}$, valeur moyenne des bornes considérées.

En l'absence de tout déconfinement, la poussée exercée sur le parement serait celle au repos et dans ce cas :

$$
T_{2}=1,5 P_{a} .
$$

Si le déconfinement est tel que l'état actif peut être atteint, alors la poussée exercée sur le parement serait :

$$
T_{2}=P_{a} .
$$

La poussée exercée sur le parement est donc :

$$
\begin{aligned}
& T_{2}=P_{a}\left[\frac{K_{o}}{K_{a}}\left(1-\frac{\delta}{\delta_{a}}\right)+\frac{\delta}{\delta_{a}}\right], \\
& \text { soit } T_{2}=P_{\mathrm{a}}\left[\frac{K_{\mathrm{o}}}{K_{\mathrm{a}}}+\frac{\delta}{\delta_{\mathrm{a}}}\left(1-\frac{K_{\mathrm{o}}}{K_{\mathrm{a}}}\right)\right],
\end{aligned}
$$

avec :

$-\delta$ : déplacement relatif mesuré lors de la réalisation de parois clouées ;

$-\delta_{\mathrm{a}}$ : déplacement relatif de la paroi clouée nécessaire pour atteindre l'état actif.

Le tableau C1 de l'annexe C3 de la norme NF 1997-1-EC7 donne les mouvements nécessaires pour atteindre l'état actif : ces derniers sont compris entre $0,05 H / 100$ et $H / 100$ suivant l'état de densité d'un sol non cohérent et du type de déplacement de la paroi clouée (Fig. 9).

Les déplacements relatifs mesurés lors de la réalisation de parois clouées sont donnés dans les recommandations CLOUTERRE (1991) et varient dans une fourchette allant de $0,2 H / 100$ à $0,4 H / 100$ pour les sols meubles (Tab. 1). 


\begin{tabular}{|l|c|c|c|}
\multicolumn{5}{|c|}{ Tableau C.1 Rapport $v_{\mathrm{a}} / \mathrm{h}$} \\
\hline \multicolumn{2}{|c|}{ Type de mouvement du mur } & $\begin{array}{c}v_{\mathrm{a}} / \mathrm{h} \\
\text { sol lâche } \\
\%\end{array}$ & $\begin{array}{c}v_{\mathrm{a}} / \mathrm{h} \\
\text { sol dense } \\
\%\end{array}$ \\
\hline a) & & 0,4 à 0,5 & 0,1 à 0,2 \\
\hline b) & & & \\
\hline & \\
\hline
\end{tabular}

Fig. 9. Tableau C1 de l'annexe C3 de la norme NF 1997-1-EC7. Fig. 9. Table C1 (appendix C3) from NF 1997-1-EC7 standard.

Une borne supérieure de $T_{2}$ est donnée pour $\delta$ minimal et $\delta_{\mathrm{a}}$ maximal, soit :

$$
\frac{\delta}{\delta_{a}}=\frac{2 H / 1,000}{0,5 H / 100}=0,4
$$

ce qui donne $T_{2}=1,3 P_{\mathrm{a}}$.

Il est donc proposé de retenir comme valeur de la poussée sur le parement :

$$
T_{2}=1,3 P_{a}
$$

De la même façon que $T_{1}, T_{2}$ est distribué entre les clous au prorata des surfaces reprises par chacun d'eux.

$\mathrm{Si}$, par contre, toute déformation était bloquée par les dispositions constructives mises en œuvre (blocage des clous à la clé dynamométrique, parois mixtes avec tirants précontraints et clous passifs...), alors la valeur proposée $T_{2}=1,3 P_{\mathrm{a}}$ sousestime la poussée appliquée sur le parement et la valeur à retenir pour $T_{2}$ est $T_{2}=1,5 P_{\mathrm{a}}=P_{0}$.

C'est pourquoi il paraît souhaitable que le concepteur puisse choisir et préciser le coefficient qui permet de passer de la poussée active calculée par la méthode de Culmann à la valeur effectivement appliquée sur le parement en fonction des déplacements prévus de la paroi.

Ce coefficient varie donc de 1,0 à 1,5 et la valeur devrait être retenue en accord avec les considérations développées dans les paragraphes précédents.

Aux fins de comparaison avec $T_{0}$ et $T_{1}$, la valeur de $T_{2}$ doit être pondérée par un coefficient de sécurité.
Tableau 1. Ordre de grandeur des déplacements en tête d'un massif cloué.

Table 1. Displacement values at top of a nailed wall.

\begin{tabular}{llll}
\hline & Sols semi-rocheux & Sables & Argiles \\
\hline$d_{\mathrm{v}}=d_{\mathrm{h}}$ & $H / 1,000$ & $2 H / 1,000$ & $4 H / 1,000$ \\
\hline
\end{tabular}

En résumé, la poussée active $P_{\mathrm{a}}$ est calculée par la méthode de Culmann sur le parement. Cette poussée active est majorée dans le rapport $K_{0} / K_{\mathrm{a}}$ pour obtenir la poussée au repos $P_{0}$ sur la même surface de calcul. La valeur de $T_{2}$ est interpolée dans les parois clouées, entre la poussée au repos à déplacement nul et la poussée active nécessitant un déplacement $\delta=0,5 \% \mathrm{H}$. Enfin, cette dernière valeur de $T_{2}$ doit être pondérée par un coefficient de sécurité.

\subsubsection{Comparaison avec la bibliographie}

L'additif 2002 aux recommandations CLOUTERRE (2002) proposait que, sur les deux premiers lits de clous, le calcul soit réalisé :

- avec $T_{2}=0,7 K_{a} \gamma h^{2}$

- soit $T_{2}=\frac{0,7}{0,5} P_{a}=1,4 P_{a}$;

- et que, sur les lits inférieurs, soit considérée la poussée active $P_{\mathrm{a}}$.

La norme NF 94-270 (Vezole et al., 2009) sur les ouvrages en sol renforcé propose que la poussée appliquée sur le parement soit la poussée active majorée sur toute la hauteur d'une contrainte horizontale constante telle que la poussée totale sur le parement soit égale à la poussée au repos, ce qui revient à considérer que $T_{2}=1,5 P_{\mathrm{a}}$ en vertu des considérations développées dans les paragraphes précédents. Cette dernière disposition paraît extrêmement sécuritaire et non conforme aux comportements et fonctionnement des ouvrages en sol renforcé.

Terzaghi et Peck (1967) ont proposé des diagrammes de pression à considérer pour le dimensionnement des blindages de fouille basés sur l'expérience qui sont :

- sables : $P=0,65 K_{\mathrm{a}} H^{2}$ soit $T_{2}=\frac{0,65}{0,5} P_{a}=1,3 P_{a}$;

- argiles molles et moyennement raides : $P=0,875$ $\left(1-\frac{4 C u}{\gamma H}\right) \gamma H^{2}$, soit $T_{2}=\frac{0,875\left(1-\frac{4 C u}{\gamma H}\right) P_{a}}{0,5}$, soit $T_{2}=1,75$ $\left(1-\frac{4 C u}{\gamma H}\right) P_{a}^{1}$

- argiles raides : $P=0,15$ à $0,30 \gamma H^{2}$, soit $T_{2}=\frac{0,15-0,30}{0,5}$ $=0,3-0,6 P_{\mathrm{a}}$.

Byrne et al. (1998) (Fig. 5.17, p. 116) ont synthétisé les valeurs d'efforts mesurés au parement. Quatre-vingts pour cent des valeurs mesurées sont telles que $P \leq 0,6 K_{\mathrm{a}} \gamma H^{2}$ avec des minimums à 0 et un maximum à $P=1,5 K_{\mathrm{a}} \gamma H^{2}$, soit $T_{2} \leq 1,2 P_{\mathrm{a}}$ pour $80 \%$ des cas et $T_{2 \mathrm{MAX}}=3 P_{\mathrm{a}}$. Ils préconisent que les efforts au niveau du parement soient considérés comme égaux à $P=0,5 K_{\mathrm{a}} \gamma H^{2}$, soit $T_{2}=1,0 P_{\mathrm{a}}$.

\footnotetext{
${ }^{1}$ Si l'on considère non pas la résistance au cisaillement non drainé $C u$ mais la cohésion effective $c$, et que l'on s'intéresse à la variation du coefficient $t=1,75\left(1-\frac{4 c^{\prime}}{\gamma H}\right)$ pour des valeurs de $\gamma$ et $H$ de $20 \mathrm{kN} / \mathrm{m}^{3}$ et $H=5 \mathrm{~m}$, la valeur de $t$ varie entre 1,75 et 1,05 pour une cohésion effective variant entre 0 et $10 \mathrm{kPa}$, soit 1,4 en moyenne.
} 
Gigan (1986) rapporte des pressions derrière le parement égales à $60 \%$ de la poussée active, soit $T_{2}=0,6 P_{\mathrm{a}}$.

Gudehus (1982), enfin, propose des valeurs $T_{2}$ variant de 1,0 à $1,2 P_{\mathrm{a}}$.

Les valeurs proposées pour $T_{2}$ varient dans une fourchette très large, de 0,6 à $1,2 P_{\mathrm{a}}$, pour les auteurs ayant suivi ou rapporté des expérimentations de parois clouées.

CLOUTERRE (1991, 2002), fondé sur l'expérimentation, et la norme NF P 94-270 (Vezole et al., 2009) proposent par contre des valeurs plus fortes, respectivement de 1,4 et 1,5.

$\mathrm{Au}$ vu des références bibliographiques et des développements proposés ci-avant, la valeur de 1,5 $P_{\text {a }}$ proposée dans la norme NF P 94-270 (Vezole et al., 2009) apparaît sécuritaire (ce que cette dernière reconnaît explicitement pour les sols cohérents) et la valeur proposée de 1,3 $P_{\mathrm{a}}$ apparaît être un meilleur estimatif pour les cas courants.

Toutefois, dans les sols ou roches tendres, évolutifs, gélifs, gonflants ou fortement sur-consolidés, une approche du comportement géotechnique de l'ouvrage doit justifier un autre choix.

\subsubsection{Diagramme de poussée sur le parement}

Indépendamment de la valeur de la poussée sur le parement, qui a fait l'objet de l'analyse développée au paragraphe précédent, se pose la question de la répartition des pressions sur le parement correspondant à la force de poussée.

Selon les documents et les auteurs (Delattre et Marten, 2003 ; Fau, 1987 ; Lazarte et al., 2003 ; Marche et Favre, 1999 ; Savao et al., 1995), la répartition à considérer pour distribuer la pression à partir de la valeur $T_{2}$ calculée peut varier de triangulaire (additif CLOUTERRE, 2002) à trapézoïdale (norme NF 94-270 [Vezole et al., 2009] et Terzaghi), voire rectangulaire (Terzaghi). Il est laissé la latitude au concepteur de choisir le type de distribution.

\subsection{Détermination de la longueur de scellement strictement nécessaire}

Le but est de déterminer la longueur minimale de chaque lit de clous qui permet d'assurer la stabilité de la paroi.

L'optimisation proposée suppose que la longueur des clous est toujours conditionnée par $T_{\text {MAX }}$.

Pour cela, l'ensemble des combinaisons de longueurs possibles doit être parcouru en imposant des contraintes constructives et géométriques de manière à obtenir le facteur de sécurité strictement nécessaire à assurer la stabilité.

La solution optimale correspond à celle dont la somme des produits (longueur de clous $\times$ résistance maximale de l'armature) sera minimale. Ce critère permet de minimiser le poids d'acier utilisé.

$$
\text { Solution }=\min _{n \text { Surfaces }}\left(\sum_{i=1}^{n \text { clous }} T_{\max }(i) \times L(i)\right) .
$$

Les contraintes constructives supplémentaires imposées sont que :

- les longueurs de clous restent constantes ou diminuent du haut vers le bas (Fig. 10);

- les longueurs de clous ne doivent pas être inférieures à une valeur minimale imposée par le concepteur.

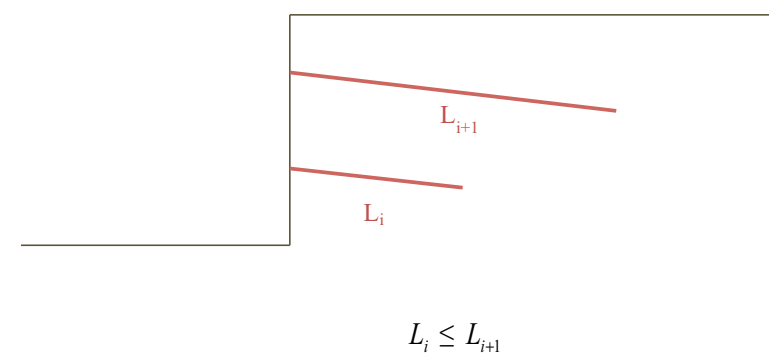

Fig. 10. Disposition constructive imposée sur la longueur des clous. Fig. 10. Constructive arrangement imposed to the length of nails.

L'algorithme d'optimisation utilise la théorie des graphes avec recherche par profondeur.

La méthode permet d'arriver rapidement à la première solution valide et d'éliminer ainsi plus facilement des itérations (Fig. 11)

Cet algorithme permet de parcourir toutes les solutions en éliminant le plus rapidement possible les «branches » que ne sont pas adéquates, c'est-à-dire ne répondant pas à la contrainte imposée ou dont la solution sera forcément moins optimale que celle trouvée précédemment.

Pour réduire le temps de calcul, il est apparu préférable d'itérer cet algorithme avec des valeurs de $\Delta L$ décroissantes, minimisant ainsi le nombre de combinaisons.

\subsection{Détermination de la section d'armature strictement nécessaire}

Connaissant l'effort $T_{\mathrm{MAX}}$ qui est strictement nécessaire pour obtenir le facteur de sécurité requis pour une surface de rupture, la section d'armature nécessaire pour la surface de rupture considérée est :

$$
A_{i}=\frac{T_{\mathrm{MAX}_{\mathrm{i}}}}{\frac{\sigma_{e}}{\Gamma \sigma_{e}}} .
$$

La section d'armature strictement nécessaire est la section maximale d'armature de toutes les surfaces de rupture :

$$
A=M A X\left[A_{i}\right] .
$$

\section{Application opérationnelle}

Les propositions développées ci-avant ont été intégrées dans un logiciel de calcul de stabilité.

L'intégration de ces propositions permet non seulement de faire une vérification du caractère admissible du confortement proposé mais une optimisation de ce dernier par une détermination des longueurs et sections optimales et des efforts amenés au parement.

Un exemple des différentes étapes et sorties en est montré sur les figures ci-dessous. Le cas présenté a, pour des raisons de clarté, été conduit à l'état limite ultime, sans coefficients de sécurité partiels, en visant un facteur de sécurité global de 1,5. La longueur initiale des clous est prise égale à $15 \mathrm{~m}$ pour une 
Lit de clous $n^{\circ} 1$

(bas de la paroi)

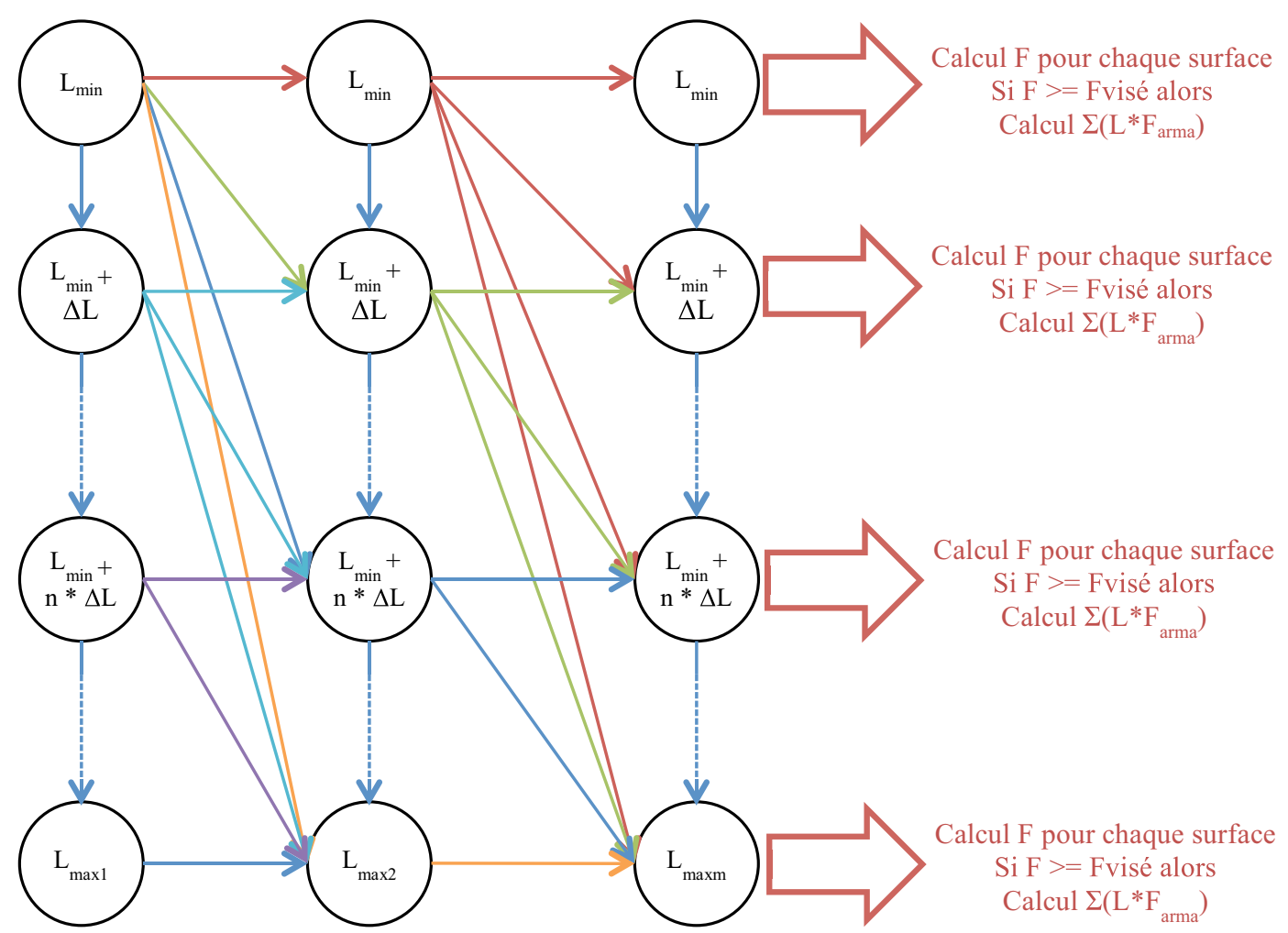

Fig. 11. Algorithme de recherche de longueurs.

Fig. 11. Algorithm to reach optimized lengths of nails.

longueur minimale imposée par le concepteur est de $5 \mathrm{~m}$. La traction élastique des clous est de $560 \mathrm{kN}$.

La Figure 12 présente les résultats du calcul donnant les longueurs optimisées pour atteindre le facteur de sécurité visé de 1,5. Ce calcul montre que, pour la longueur de clous initiale, le facteur de sécurité obtenu est de 1,772 et que l'on obtiendra un facteur de sécurité de l'ordre de 1,5 pour des clous dont la longueur est de $11,35 \mathrm{~m}$ pour tous les clous.

La Figure 13 présente le facteur de sécurité calculé dans le cas où la longueur des clous est effectivement de $11,35 \mathrm{~m}$. Le résultat est celui attendu, le facteur de sécurité obtenu est égal à 1,502. Dans ce calcul, les efforts $T_{\mathrm{MAX}}, T_{0}, T_{1}$ et $T_{2}$ tels que définis dans les paragraphes précédents obtenus sont également calculés. Ils montrent que la traction maximale dans les clous $T_{\mathrm{MAX}}$ est comprise entre 47 et $164 \mathrm{kN}$. Au niveau du parement, les efforts maximums sont principalement conditionnés par $T_{2}$, les valeurs étant comprises entre 70 et $150 \mathrm{kN}$. Ces valeurs étant supérieures à $T_{\mathrm{MAX}}$ pour certains lits, une vérification supplémentaire de la longueur devrait être réalisée. Ce point fera l'objet d'améliorations de l'algorithme.

La Figure 14 présente le facteur de sécurité obtenu en imposant les résistances résultant du calcul de $T_{\mathrm{MAX}}$ présenté sur la Figure 13. Les résultats obtenus sont conformes aux attentes, le facteur de sécurité n'ayant pas évolué malgré la diminution de la résistance de la barre $(F=1,502)$.
Le calcul de $T_{\mathrm{MAX}}$ étant réalisé pour toutes les surfaces de rupture, il est possible d'en récupérer la valeur maximale sur l'ensemble des surfaces, mais aussi le lieu où cette valeur est calculée, qui correspond à l'intersection entre la surface $S_{\mathrm{i}}$ et le clou $T_{\mathrm{i}}$, constituant le lieu des $T_{\mathrm{MAX}}$. La Figure 15 présente ainsi le lieu des efforts maximums dans les clous.

Le diagramme de poussée active calculé par la méthode de Culmann est montré sur la Figure 16.

La Figure 17 présente le diagramme de pression déduit du choix du maximum $T_{0} ; T_{1}$ et $T_{2}$ redistribué sur le parement.

\section{Conclusions}

À partir d'un constat de la situation et d'un état de l'art portant sur le dimensionnement des parois clouées, il a été proposé des méthodes permettant de déterminer les longueurs et sections minimales d'armatures, d'optimiser le dimensionnement des inclusions et de déterminer les efforts ponctuels appliqués au parement par les têtes d'inclusions. L'application opérationnelle qui en a été faite s'avère permettre une optimisation économique intéressante du dimensionnement.

Il est recommandé de confronter cette optimisation à une étude de sensibilité du dimensionnement à une variation des paramètres prépondérants que sont l'angle de frottement et le frottement latéral unitaire limite. 


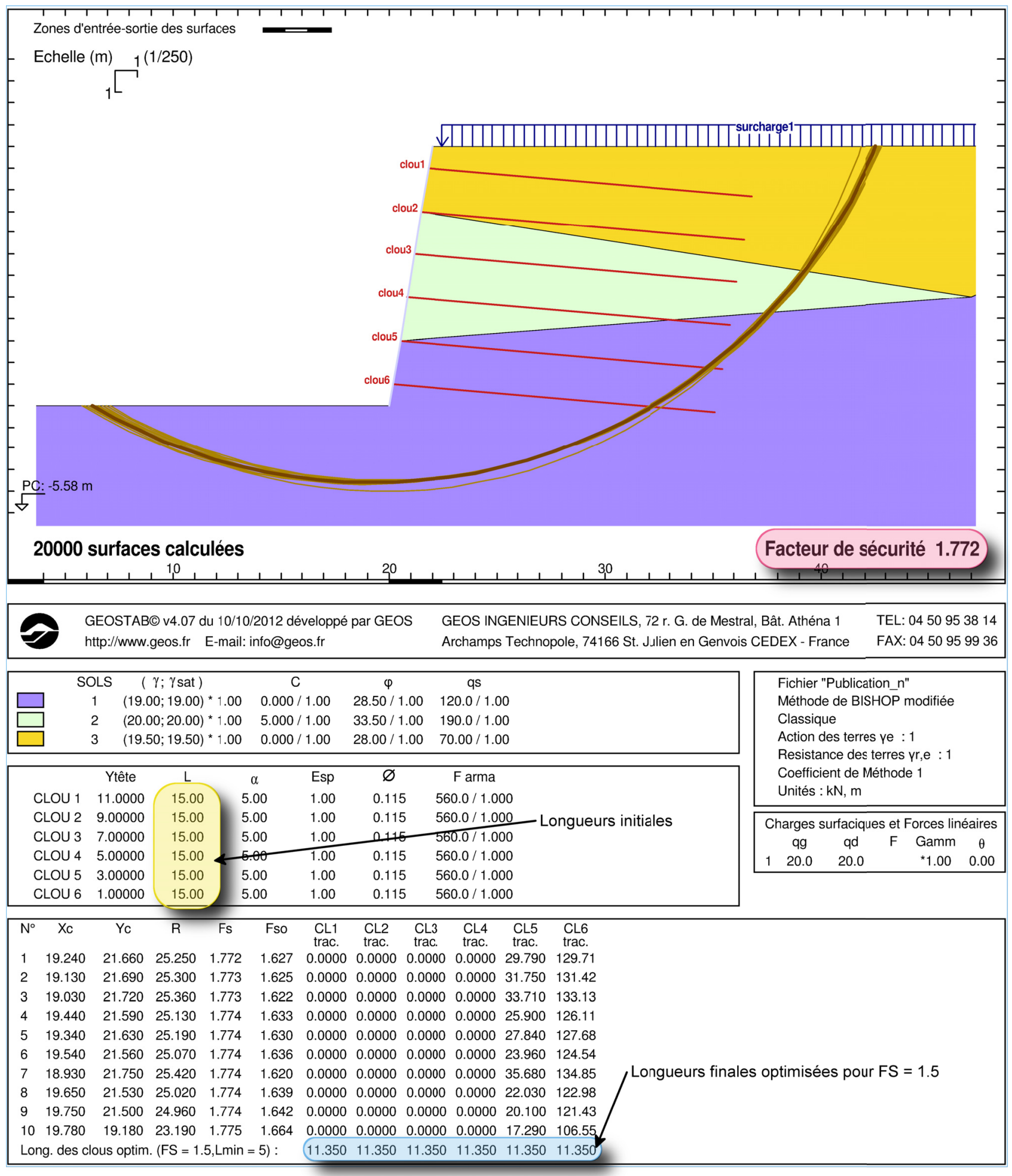

Fig. 12. Calcul de la longueur optimisée pour atteindre un $F_{\text {visé. }}$

Fig. 12. Determination of the optimized length of the reinforcement to reach the safety factor. 


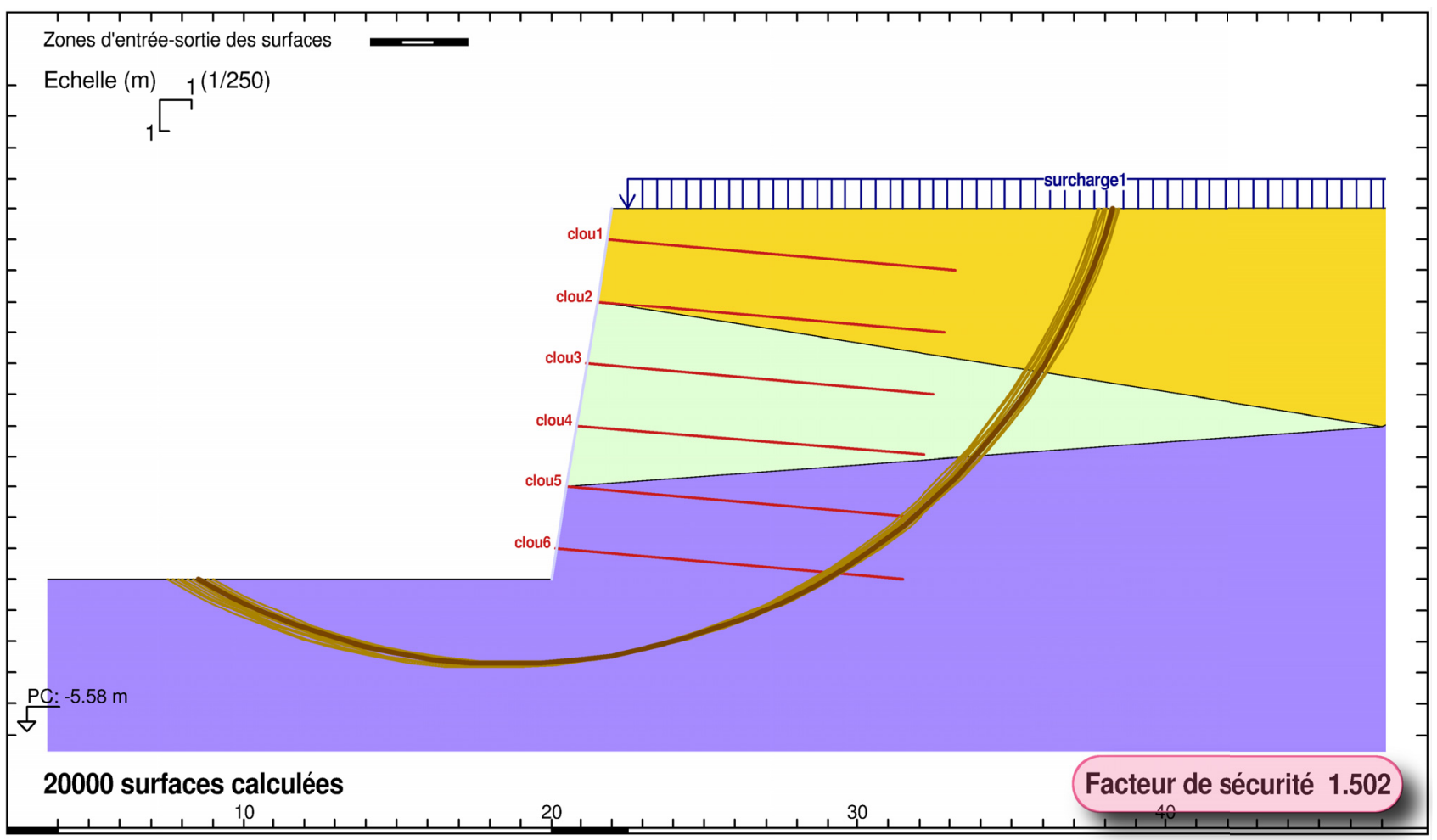

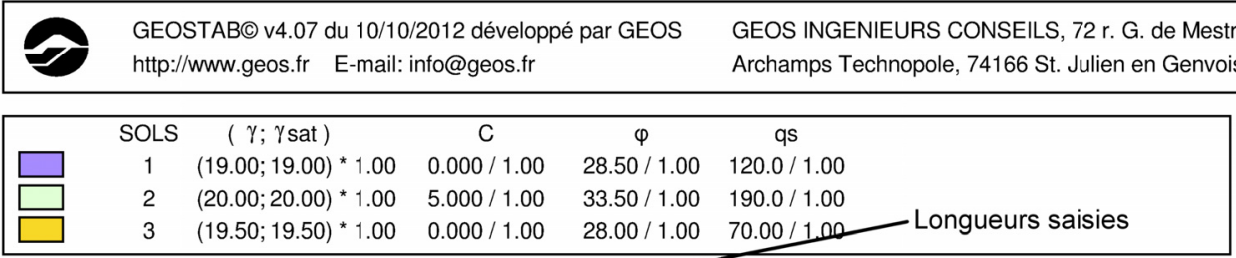

\begin{tabular}{|c|c|c|c|c|c|c|c|}
\hline & Ytête & L & $\alpha$ & Eop & $\varnothing$ & F arma & \\
\hline CLOU 1 & 11.0000 & 11.35 & 5.00 & 1.00 & 0.115 & $560.0 / 1.000$ & \\
\hline CLOU 2 & 9.00000 & 11.35 & 5.00 & 1.00 & 0.115 & $560.0 / 1.000$ & Résistances à la \\
\hline CLOU 3 & 7.00000 & 11.35 & 5.00 & 1.00 & 0.115 & $560.0 / 1.000$ & traction initiales \\
\hline CLOU 4 & 5.00000 & 11.35 & 5.00 & 1.00 & 0.115 & $560.0 />000$ & \\
\hline CLOU 5 & 3.00000 & 11.35 & 5.00 & 1.00 & 0.115 & $560.0 / 1.000$ & \\
\hline CLOU 6 & 1.00000 & 11.35 & 5.00 & 1.00 & 0.115 & $560.0 / 1.000$ & \\
\hline
\end{tabular}

Méthode de BISHOP modifiée

Classique

Action des terres ye : 1

Resistance des terres $\gamma r, \mathrm{e}: 1$

Coefficient de Méthode 1

Unités : kN, m

Charges surfaciques et Forces linéaires

99 qd F Gamm $\theta$

$\begin{array}{lllll}1 & 20.0 & 20.0 & * 1.00 & 0.00\end{array}$

\begin{tabular}{|c|c|c|c|c|c|c|c|c|c|c|c|c|c|}
\hline $\mathrm{N}^{\circ}$ & Xc & Yc & $\mathrm{R}$ & Fs & Fso & $\begin{array}{l}C L 1 \\
\text { trac. }\end{array}$ & $\begin{array}{l}\mathrm{CL} 2 \\
\text { trac. }\end{array}$ & $\begin{array}{l}\text { CL3 } \\
\text { trac. }\end{array}$ & $\begin{array}{l}\mathrm{CL} 4 \\
\text { trac. }\end{array}$ & $\begin{array}{l}\text { CL5 } \\
\text { trac. }\end{array}$ & $\begin{array}{l}\text { CL6 } \\
\text { trac. }\end{array}$ & $\underset{\text { trac. }}{\Sigma}$ & \\
\hline 1 & 18.710 & 17.610 & 20.350 & 1.502 & 1.409 & 0.0000 & 0.0000 & 0.0000 & 0.0000 & 1.7500 & 97.390 & 99.140 & \\
\hline 2 & 18.600 & 17.630 & 20.380 & 1.502 & 1.404 & 0.0000 & 0.0000 & 0.0000 & 0.0000 & 4.8200 & 100.09 & 104.91 & Efforts maximums dans les inclusions \\
\hline 3 & 18.830 & 17.600 & 20.310 & 1.503 & 1.414 & 0.0000 & 0.0000 & 0.0000 & 0.0000 & 0.0000 & 94.690 & 94.690 & 'ensemble des surfaces analysees \\
\hline 4 & 18.480 & 17.640 & 20.420 & 1.503 & 1.399 & 0.0000 & 0.0000 & 0.0000 & 0.0000 & 7.9000 & 102.81 & 110.71 & \\
\hline 5 & 18.360 & 17.660 & 20.460 & 1.504 & 1.395 & 0.0000 & 0.0000 & 0.0000 & 0.0000 & 10.990 & 105.53 & 116.52 & \\
\hline 6 & 18.250 & 17.680 & 20.490 & 1.505 & 1.391 & 0.0000 & 0.0000 & 0.0000 & 0.0000 & 14.090 & 108.27 & & \\
\hline 7 & 18.940 & 17.580 & 20.270 & 1.506 & 1.419 & 0.0000 & 0.0000 & 0.0000 & 0.0000 & 0.0000 & 92.010 & 92.910 & \\
\hline 8 & 18.130 & 17.690 & 20.530 & 1.507 & 1.387 & 0.0000 & 0.0000 & 0.0000 & 0.0000 & 17.190 & 111.01 & $12 \% .20$ & \\
\hline 9 & 18.010 & 17.710 & 20.570 & 1.508 & 1.383 & 0.0000 & 0.0000 & 0.0000 & 0.0000 & 20.310 & 113.77 & 134.08 & \\
\hline 10 & 19.060 & 17.570 & 20.240 & 1.508 & 1.424 & 0.0000 & 0.0000 & 0.0000 & 0.0000 & 0.0000 & 89.340 & 89.340 & \\
\hline \multicolumn{6}{|c|}{ Efforts maximums dans les clous $(\mathrm{FS}=1.5)$ : } & 47.629 & 47.982 & 137.13 & 156.24 & 121.22 & 163.89 & 674.09 & \\
\hline \multicolumn{6}{|c|}{ Efforts T0 $(\mathrm{FS}=1.5)$ : } & 3.1660 & 2.4330 & 0.0000 & 14.309 & 48.866 & 105.10 & 173.88 & forts maximums au parement \\
\hline \multicolumn{6}{|c|}{ Efforts $\mathrm{T} 1(\mathrm{FS}=1.5):$} & 23.133 & 23.133 & 2.9807 & 2.9807 & 2.9807 & 22.624 & & " \\
\hline \multirow{2}{*}{\multicolumn{6}{|c|}{$\begin{array}{l}\text { Efforts } \mathrm{T} 2(\mathrm{FS}=1.5 ; \mathrm{T} 2 / \mathrm{Pa}=1.3 ; \delta / \varphi=0) \text { : } \\
\text { Maximums } \mathrm{T} 0, \mathrm{~T} 1, \mathrm{~T} 2:\end{array}$}} & 70.052 & 149.31 & 149.64 & 149.64 & 149.14 & 68.954 & 736.73 & \\
\hline & & & & & & 70.052 & 149.31 & 149.64 & 149.64 & 149.14 & 105.10 & & \\
\hline
\end{tabular}

Fig. 13. Calcul des efforts maximums dans les inclusions $T_{\mathrm{MAX}}$, et des efforts appliqués au parement $T_{0}, T_{1}, T_{2}$.

Fig. 13. Determination of the maximum efforts in the nail bars $T_{M A X}$, and of the efforts applied to the face $T_{0}, T_{1}, T_{2}$. 


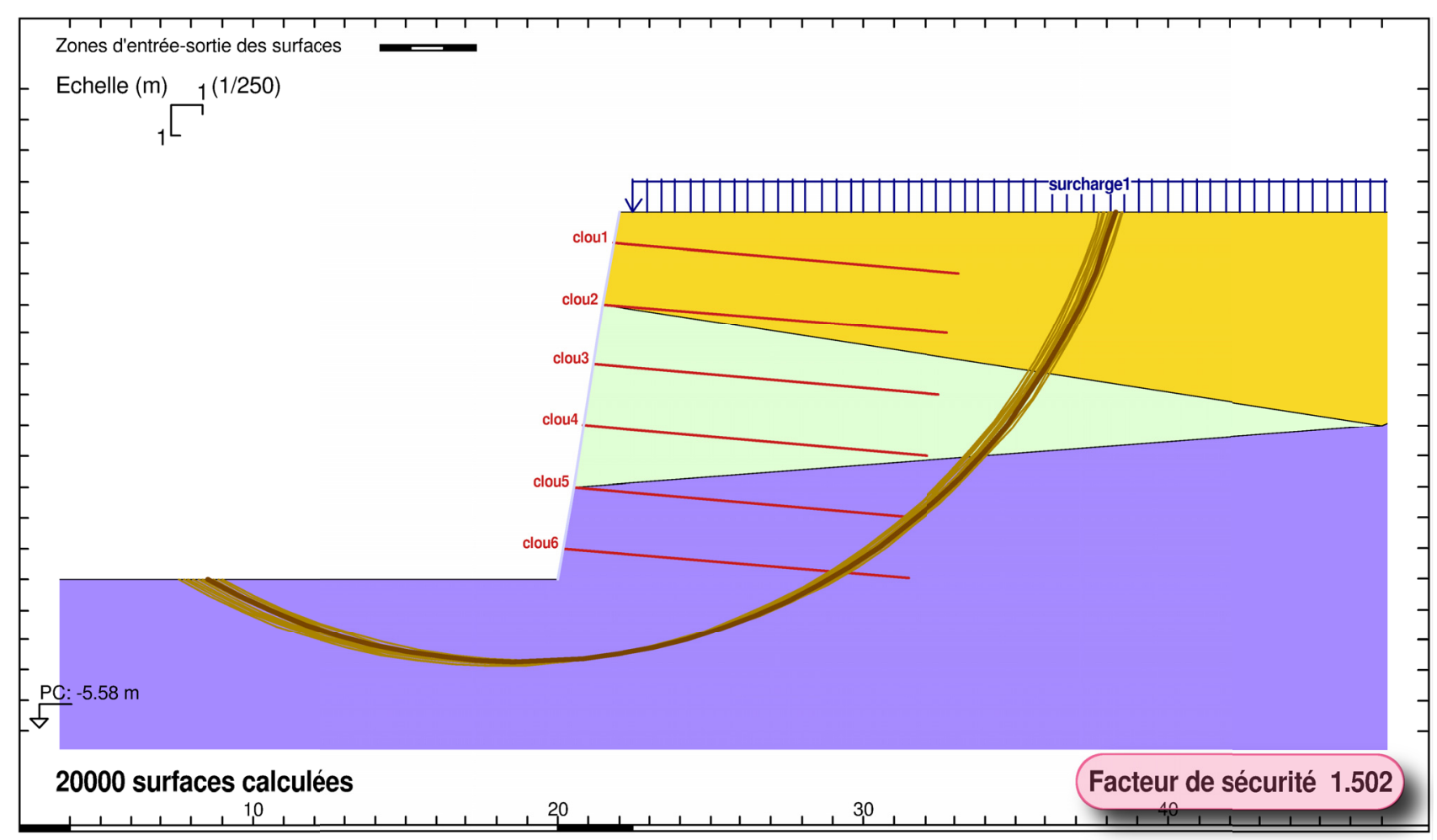

\begin{tabular}{|c|c|c|c|c|c|}
\hline & \multicolumn{4}{|c|}{$\begin{array}{l}\text { GEOSTAB@ v4.07 du 10/10/2012 développé par GEOS } \\
\text { http://www.geos.fr E-mail: info@geos.fr }\end{array}$} & $\begin{array}{l}\text { GEOS INGENIEURS CONSEILS, } 72 \text { r. G. de Mestl } \\
\text { Archamps Technopole, } 74166 \text { St. Jalien en Genvoi }\end{array}$ \\
\hline & SOLS & $(\gamma ; \gamma$ sat $)$ & $\mathrm{C}$ & $\varphi$ & qs \\
\hline & 1 & $(19.00 ; 19.00) * 1.00$ & $0.000 / 1.00$ & $28.50 / 1.00$ & $120.0 / 1.00$ \\
\hline & 2 & $(20.00 ; 20.00) * 1.00$ & $5.000 / 1.00$ & $33.50 / 1.00$ & $190.0 / 1.00$ \\
\hline & 3 & $(19.50 ; 19.50) * 1.00$ & $0.000 / 1.00$ & $28.00 / 1.00$ & $70.00 / 1.00$ \\
\hline
\end{tabular}

\begin{tabular}{|lccccccc|}
\hline & Ytête & $\mathrm{L}$ & $\alpha$ & Esp & $\varnothing$ & F arma & \\
CLOU 1 & 11.0000 & 11.35 & 5.00 & 1.00 & 0.115 & $47.63 / 7.000$ & Résistance à la traction \\
CLOU 2 & 9.00000 & 11.35 & 5.00 & 1.00 & 0.115 & $47.98 / 1.000$ & saisie correspondant \\
CLOU 3 & 7.00000 & 11.35 & 5.00 & 1.00 & 0.115 & $137.1 / 1.0 \varnothing 0$ & aux efforts maximums \\
CLOU 4 & 5.00000 & 11.35 & 5.00 & 1.00 & 0.115 & 156.2 & de la page précédente \\
CLOU 5 & 3.00000 & 11.35 & 5.00 & 1.00 & 0.115 & $121.2 / 1.000$ & \\
CLOU 6 & 1.00000 & 11.35 & 5.00 & 1.00 & 0.115 & $163.9 / 1.000$ & \\
\hline
\end{tabular}

TEL: 0450953814 http://www.geos.fr E-mail: info@geos.f FAX: 0450959936

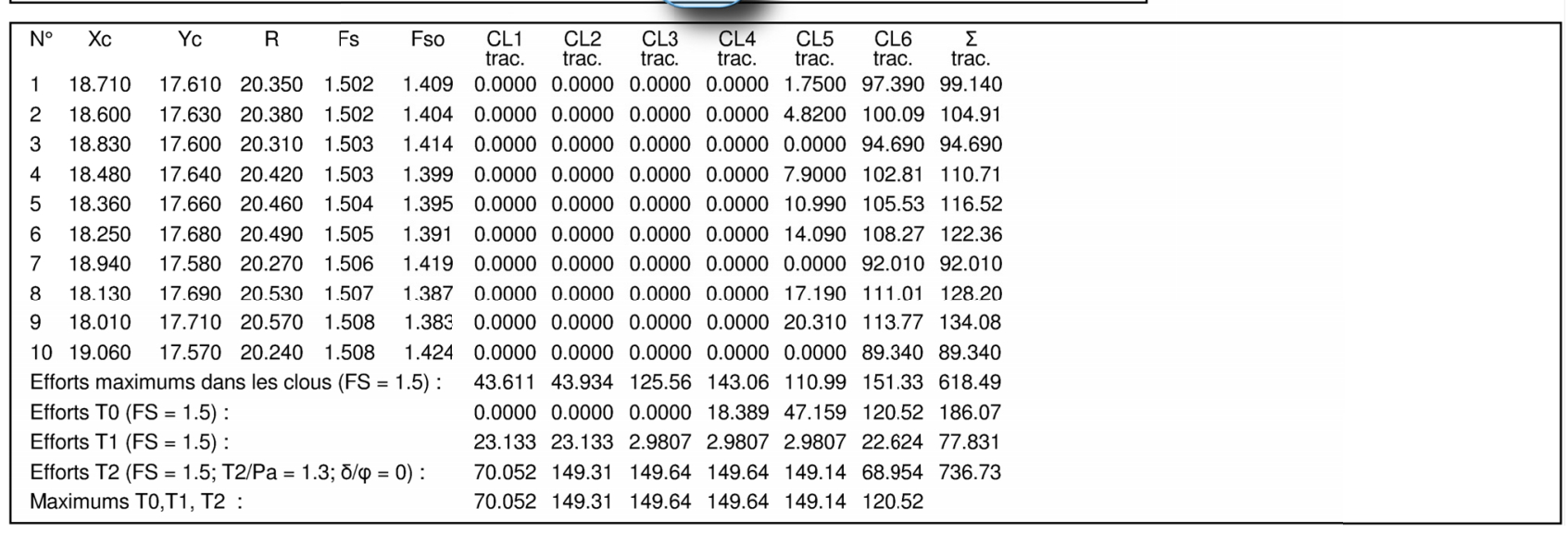

Fig. 14. Vérification du calcul des efforts maximums $T_{\mathrm{MAX}}$ dans les inclusions.

Fig. 14. Checking of the maximal efforts $T_{M A X}$ in the nails. 


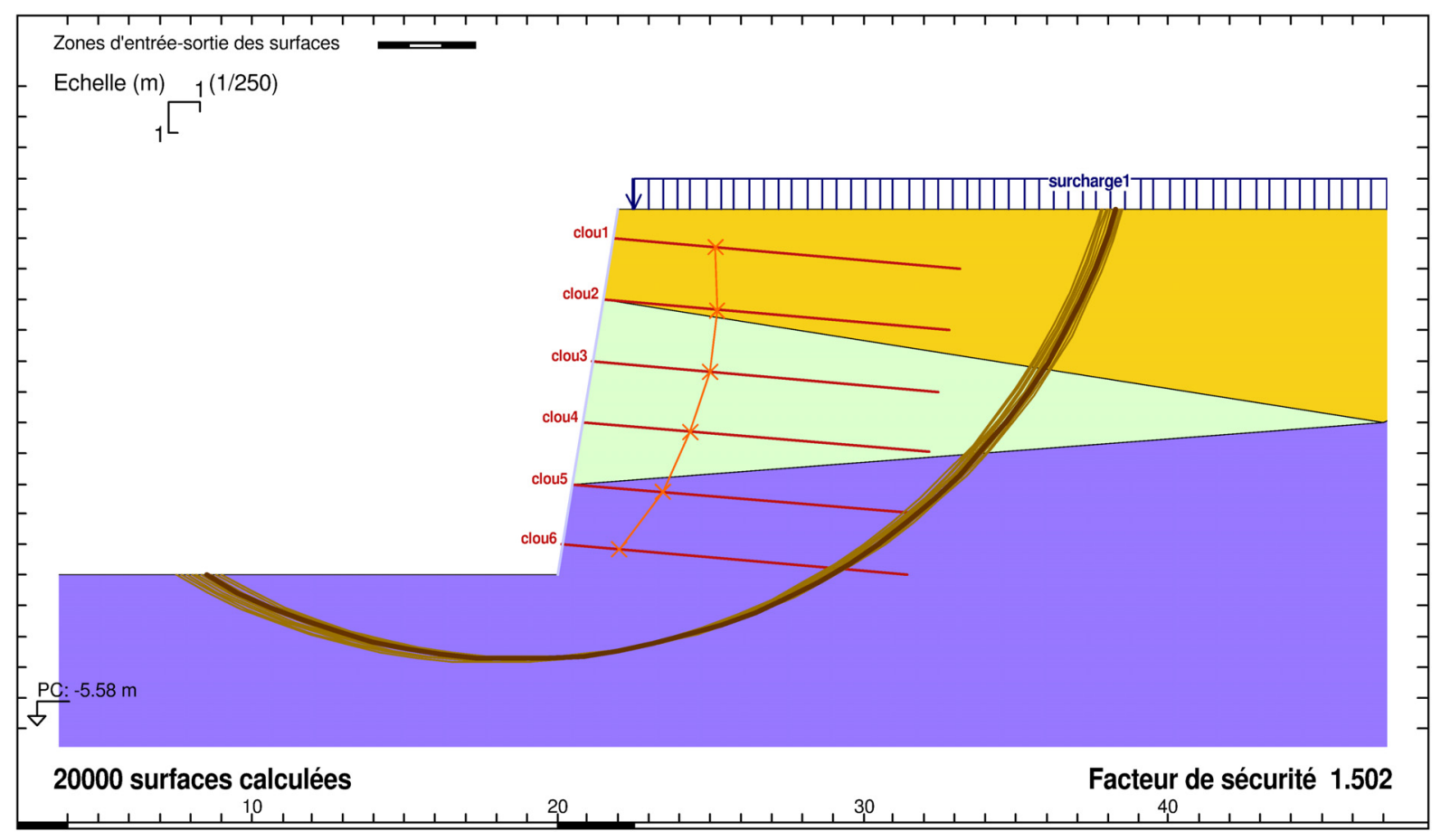

Fig. 15. Lieux des efforts maximums dans les inclusions.

Fig. 15. Location of the maximum efforts in the nails.

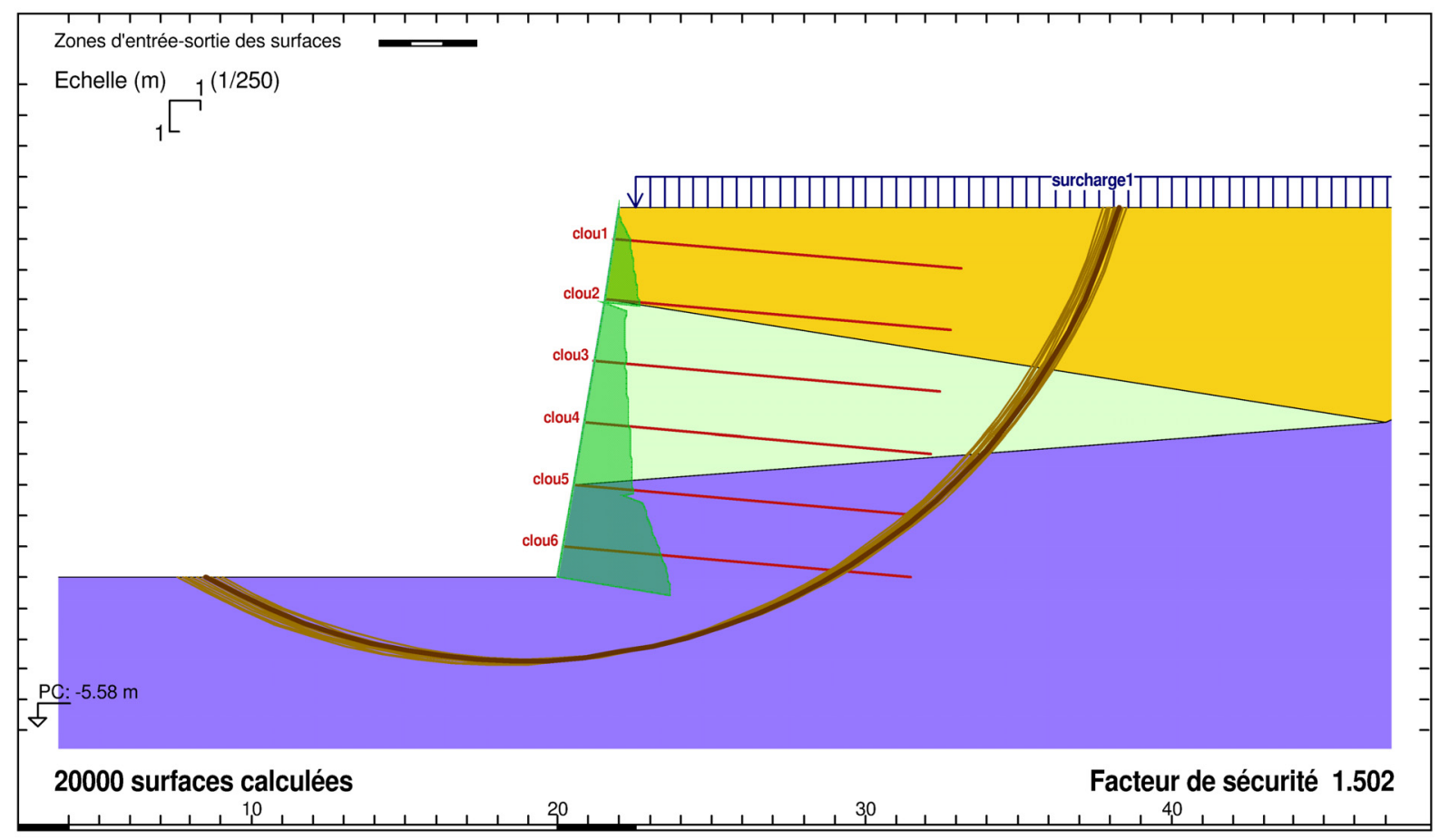

Fig. 16. Diagramme des pressions appliquées au parement calculées par la méthode de Culmann.

Fig. 16. Diagram of pressure applied to the face according to Culmann's method. 


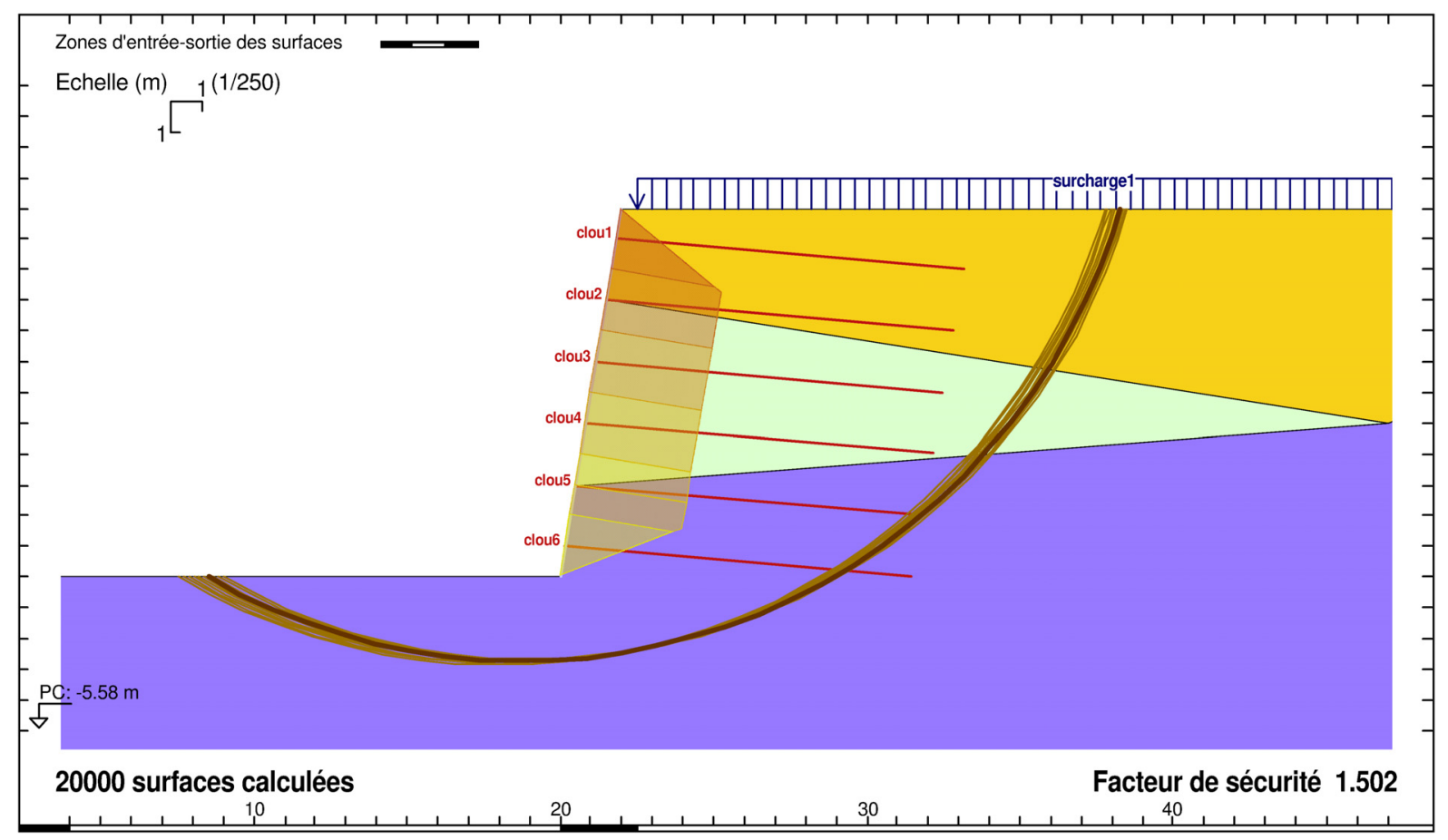

Fig. 17. Diagramme de pressions redéfini pour le calcul de l'effort $T_{2}$.

Fig. 17. Diagram of distributed pressure for the determination of the applied pressure to the face.

Les cas particuliers des sols gonflants, gélifs, évolutifs doivent faire l'objet d'une étude particulière pour déterminer les pressions appliquées au parement.

Remerciements. Les auteurs tiennent à remercier messieurs C. Chapeau et A. Ménoret (Chapeau, 2012 ; Ménoret, 2012) qui ont bien voulu nous faire part de leurs critiques constructives, avis, conseils et remarques éclairées après des relectures minutieuses.

\section{Références}

Blondeau F, et al. 1984. Méthode de calcul des ouvrages en terre renforcée - Renforcement en place des sols et des roches. In : Comptes rendus du colloque international. Presse de l'ENPC, Paris, 9-11 octobre, 1984.

Byrne RJ, et al. 1998. Manual for design and construction monitoring of soil nail walls. In: Report FHWA-SA-96-69R.

Chapeau C. 2012. Communications personnelles 2011.

CLOUTERRE. 1991. Recommandations CLOUTERRE 1991. Presses de l'ENPC.

CLOUTERRE. 2002. Additif aux recommandations CLOUTERRE 1991. Presses de l'ENPC.
Delattre L, Marten S. 2003. Un siècle de calcul d'écran de soutènement. Bull Lab Ponts Chaussees 244-245: 31-51.

Fau D. 1987. Le clouage des sols - Application au soutènement de fouille - Étude expérimentale et dimensionnement. Thèse ENPC.

Gigan JP, 1986. Applications du clouage en soutènement - Paramètres de conception et de dimensionnement des ouvrages. Bull Liaison Ponts Chaussees 143: 51-64.

Gudehus G. 1982. Clouage des sols - règles de dimensionnement et leur vérification expérimentale. Rev Fr Geotech 19: 24-37.

Lazarte CA, et al. 2003. Geotechnical engineering circular No. 7 soil nail walls. In: Report FHWA, DTEH61-00-C-00109.

Marche M, Favre M. 1999. Dimensionnement et fluage à moyen terme d'un mur cloué de grande hauteur. In : Conférence Société suisse de mécanique des sols et des roches, Berne, 23 avril 1999.

Ménoret A. 2012. Communications personnelles 2011.

Sayao ASFJ, et al. 1995. Aspects de projet et instrumentation d'un talus cloué de $40 \mathrm{~m}$ de hauteur. In: International Conference on Soil Mechanics and Geotechnical Engineering, Osaka, septembre 1995.

Terzaghi K, Peck EB. 1967. Soil mechanics in engineering practice, 2nd ed. New York: John Wiley \& Sons.

Tschebotarioff GP. 1968. Chapitre 5 dans les fondations G.A. Leonards. Ouvrages de soutènement. Dunod Éditeur.

Vezole P, et al. 2009. Calcul géotechnique - Ouvrages de soutènement - Remblais renforcés et massifs en sol cloué NF P 94-270AFNOR.

Citation de l'article : Marc Favre, Éric Vermoote, Damien Fuentes. Réflexions et propositions pour le calcul optimisé des parois clouées. Rev. Fr. Geotech. 2017, 150, 1. 